\title{
Ontology Based Business Simulations
}

\author{
Thomas Farrenkopf ${ }^{1}$, Michael Guckert ${ }^{1}$, Neil Urquhart $^{2}$, Simon
Wells $^{2}$ \\ ${ }^{1}$ KITE - Kompetenzzentrum für Informationstechnologie, Technische Hochschule Mittelhessen, Wilhelm- \\ Leuschner-Straße 13, Friedberg 61169, Germany \\ ${ }^{2}$ School of Computer, Edinburgh Napier University, Merchiston Campus, 10 Colinton Road, Edinburgh EH10 \\ 5DT, United Kingdom \\ Correspondence should be addressed to thomas.farrenkopf@mnd.thm.de \\ Journal of Artificial Societies and Social Simulation 19(4) 14, 2016 \\ Doi: 10.18564/jasss.3266 Url: http://jasss.soc.surrey.ac.uk/19/4/14.html \\ Received: 13-07-2016 Accepted: 03-10-2016 Published: 31-10-2016
}

\begin{abstract}
Within business games there is a need to provide realistic feedback for decisions made, if such business games are to continue to remain relevant in increasingly complex business environments. We address this problem by using software agents to simulate individuals and to model their actions in response to business decisions. In our initial studies we have used software agents to simulate consumers who make buying decisions based on their private preferences and those prevalent within their social network. This approach can be applied to search for behavioural patterns in social structures and to verify predicted values based on a priori theoretical considerations. Individual behaviour can be modelled for each agent and its effects within the marketplace can be examined by running simulations. Our simulations are founded upon the BDI software model (belief-desire-intention) combined with ontologies to make world knowledge available to the agents which can then determine their actions in accordance with this knowledge. We demonstrate how ontologies can be integrated into the BDI concept utilising the Jadex agent framework. Our examples are based upon the simulation of market mechanisms within the context of different industries. We use a framework, developed previously, known as AGADE within which each agent evolves its knowledge using an ontology maintained during the simulation. This generic approach allows the simulation of various consumer scenarios which can be modelled by creating appropriate ontologies.
\end{abstract}

Keywords: Social Simulation, Ontology, BDI Agent

\section{Introduction}

\section{Motivation}

1.1 Business games are an important tool for teaching students the consequences of decision making within a business context. In a business game environment players determine sequences of activities e.g. producing a certain quantity of products with a given set of features, a pricing strategy or a marketing budget. Decisions are governed by constraints within the game scenario such as restricted budget or limited production capacities. The business game then evaluates the input of the players and presents feedback detailing the effect of the decision. Such feedback will typically be an estimate of the quantity of products sold, the market share and the resulting profit/loss. The players take turns to interact with this model over a number of iterations in which the model computes likely sales figures for the product portfolios offered by the companies under simulation.

\section{Problem}

1.2 Simulation of real world scenarios in business games is an important application of complex adaptive social systems. These complex adaptive systems will gain more and more importance and the science of complexity will become one of the core disciplines of the future. Current business games do not give sufficient feedback to 
the participants to draw conclusions on cause and effect. The underlying models are based on sets of difference or differential equations (see Forrester 1990, in his System Dynamics approach) or other methods that emphasise quantitative aspects (see Gros 2008). Agents are a far more natural instrument to model complex dynamic systems as they allow to directly model individual behaviour which in consequence leads to system wide effects. A restructuring of the representational infrastructure of complex social systems has occurred as Wilensky $\&$ Rand 2015 put forth and this will in consequence have an impact on the quality of business simulations.

\section{Idea}

1.3 The effectiveness of a business game as an educational tool depends entirely on its feasibility to real world scenarios and on the instant feedback provided by the game which should both appear as realistic as possible (see Kaynak et al. 2012; Baldissin et al.2013). The more realistic the model the more valuable the educational experience. In our scenario we assume that the business game involves specifying, producing and pricing a consumer product which is then released into a marketplace for sale. Products perceived as desirable will outsell those perceived as less attractive. We propose that realistic simulation of a consumer marketplace can be achieved by the use of agents to model consumer behaviour and communication.

1.4 According to classical definitions, agent-based models consist of agents that are autonomous computational individuals interacting with each other and their environment. They are active as well as reactive: external stimuli, internal state and available information are used to determine actions an agent performs to reach given goals. Agents are particularly well suited to represent social actors in complex environments which we use as a basis for our approach to build business games.

1.5 A conceptual framework that guides agent activities is the BDI model which is a well established paradigm for the development of agents (Bratman 1999). A BDI agent has knowledge about its world (beliefs) and pursues goals (desires) while following given strategies (intentions). Therefore, the agent's belief base stores everything an agent knows (or believes to know) about the environment it lives and acts in. This knowledge is used to model an image of the relevant world with all required elements that exist and how these elements are related to each other.

\section{Contribution}

1.6 Agents must be able to act according to their knowledge of their internal and external world which must therefore be expressed in an appropriate format. Standardised formal languages to represent knowledge are available now and can be used to model knowledge of an agent in so called ontologies. They can be used to equip agents acting in a simulation with personalised knowledge and learning capabilities. The idea of supporting communities of agents with ontologies which describe the objects involved in the interactions of the agents was originally formulated by Gruber (1993) and has subsequently been discussed by various other authors (e.g. Hadzic 2009). Currently, frameworks mostly just perform syntactic matchings to detect analogies between data exchanged during the communication process of a simulation (Hadzic 2009, pp. 1-35). Strong and well designed connections between semantic technologies and multi-agent systems can have a significant effect on the quality of multi-agent simulations.

1.7 By using a community of agents, we propose that the simulated market place can be made more realistic in its feedback to player inputs. By using a complex structure with many agents making individual decisions based on local criteria and the influence of other local agents, the responses should be sufficiently complex to model market place behaviour. This complexity should encourage the student to focus on making realistic business decisions instead of simply learning the game mechanisms.

1.8 A major benefit of supplying agents with ontologies in modelling simulation scenarios is a shift in perspective that separates modelling aspects from coding details and focusses on the model rather than on programming details. This increases efficiency and leads to a clearer separation of concerns.

1.9 The contribution of this work is a framework that incorporates semantic technologies, network analysis algorithms and agents to run business scenarios. Agile Agent Development Environment (AGADE) shows a practical integration of a multi-agent based system and semantic technologies for realistic modelling of individuals participating in a dynamic market environment. It has been used to run simulations on a model of a mobile phone market in which buyers base their buying decision on social influence. Therefore the agents were modelled to follow the pattern of opinion leadership and the market development indeed developed as predicted and produced the expected outcome Farrenkopf et al.|2014. Additional behavioural patterns are presented in Farrenkopf et al. 2015. 


\section{Outline of the paper}

1.10 In Section 2 we describe the multi-agent simulation tool AGADE that incorporates semantic technologies and network analysis algorithms. It is a highly configurable tool that can run BDI agent simulations where agents communicate with each other, have knowledge of their environment and act in structured social environments Farrenkopf et al. 2014). The combination of BDI agents and ontologies creates a new perspective for multiagent simulation scenarios by modelling agent knowledge with the help of semantic technologies. AGADE has accomplished the integration of these semantic technologies and agent based systems following the BDI pattern. We describe the integration of ontologies with BDI agents. For the development of a universally applicable integration of semantic technologies and agent based systems the blueprint of a layered ontology architecture is demonstrated.

1.11 In Section 3 we describe the process of applying the underlying AGADE mechanisms to a business game scenario. The scenario models the introduction of a new product into an existing marketplace, namely biofuel into the saturated motor fuel market of Austria. We compare the results obtained using AGADE with simulation results published earlier. Subsequently, we allow two student groups to participate and assess their experience when using the simulation. Section 4 presents conclusions and future work.

\section{Agents and Ontologies}

2.1 In classical implementations of multi-agent systems following the BDI architectural pattern built with frameworks such as Jadex, all aspects have to be coded using a conventional programming language (mostly Java). AGADE also uses Jadex, being Java based it could therefore be seamlessly connected to the reasoning mechanisms of the ontologies. While the basic operations of agents are left in Java classes, we shift certain aspects of the $\mathrm{BDI}$ agent into the ontology so that we can use declarative rule languages such as the ontology language OWL (Web Ontology Language, W3C OWL Working Group 2012). This leads to a strict separation of concerns where epistemological definitions and rules can be separated from behavioural patterns which will still be implemented in the underlying BDI framework. AGADE makes use of well established semantic technologies and connects them to the BDI machinery. The use of ontologies serves two purposes in our approach: according to Gruber's classic postulate (1993) it defines a common vocabulary for communication and beyond that it allows an abstraction of certain elements of the simulation. For example, simulating market mechanisms with typical actors is structurally equivalent for different markets. They differ in detail only e.g. in the goods being traded and specific behavioural patterns. Our approach described in detail below uses a three layer structure to exploit these similarities.

2.2 OWL is a standardised general knowledge representation language in which we formulate what is called an ontology. Ontologies are used to declare facts (i.e. structural knowledge) and to define how conclusions can be drawn (i.e. inference knowledge) by using available reasoning instruments. Formally, an ontology $\mathcal{O}$ is a triple $(\mathcal{C}, \mathcal{R}, \mathcal{I})$ where $\mathcal{C}$ is a set of concepts, $\mathcal{R}$ a set of relations, and $\mathcal{I}$ a set of individual objects. Concepts formally denote subsets of objects. From a perspective of formal logic such subsets are the extension of concepts itself while concepts define the intentional representation of the corresponding set of objects. An object that belongs to a concept is called an instance of that concept. The elements of $\mathcal{R}$ are relations (also called roles or object properties, as they manifest the linking on the level of the instances) having subsets of $\mathcal{C}$ as domain and range. The extension of a role is then a set of pairs $(c, d)$ with $c, d \in \mathcal{I}$. Additionally, instances can have data properties through which they get linked to primitive data e.g. strings or numbers. Typically, ontologies are formulated by means of description logics with differing levels of expressiveness (Baader 2003). Usually description logics are proper subsets of first order logic where expressiveness has been traded for decidability. Inference knowledge is implicitly given by the underlying mechanisms of the available reasoning instruments. Here, we use OWL and the rule language SWRL (Semantic Web Rule Language) both specified by W3C (Horrocks et al. 2004; W3C OWL Working Group 2012). Basically, SWRL is a tool used to define if-then rules expressed in terms of OWL concepts.

2.3 Each agent is equipped with a personal reasoner and a private ontology which is accessed using the OWL API Horridge \& Bechhofer 2011). Social aspects and information about internal aspects of the agent (e.g. its current state) are mapped to the ontology (see Figure 11: the set of beliefs (i.e. knowledge), desires (i.e. goals) and intentions (i.e. plans of how to reach the goals). Factual knowledge of the world of an agent is represented by individuals and corresponding relations. Relations can be restricted by specifying domain and range. Data properties link concepts and individuals to primitive data e.g. strings or numbers (i.e. the age of a person). 


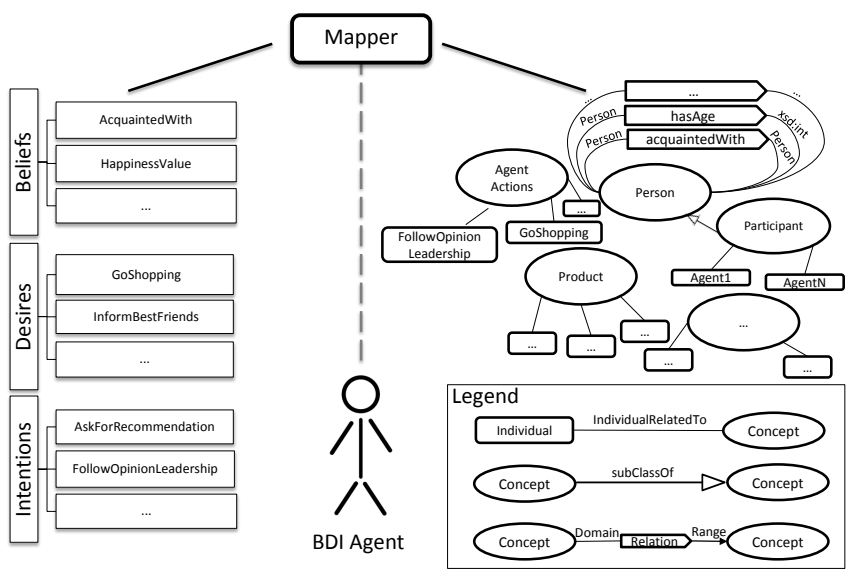

Figure 1: OWL-BDI-Mapping

2.4 We propose a layered ontology in which domain knowledge can be hierarchically separated by its degree of generality. Abstract general concepts (e.g. thing, person,...) are coded in the ADL (abstract domain layer) while more specific elements (e.g. concepts of a certain market theory) belong to the SDL (specific domain layer). Finally, individual beliefs and desires are included in the IDL (individual domain layer). While ADL and SDL are shared by all agents and therefore ensure ontological commitment without which communication between the agents would not be possible, IDL is private to each agent and thus will usually differ from agent to agent. Figure 2 shows the three layers and ontologies are imported. Each IDL concept has to be a subconcept of one ore more concepts defined either in SDL or ADL. As OWL allows ontologies to import other ontologies we can use this to include the general ontologies into the more specific ones. From a mathematical point of view the set of general concepts is a subset of the specific knowledge available to an agent. By separating knowledge into layers, general control of the simulation is kept independent of specific terms of a given scenario. During the process of communication, agents can exchange elements of their IDLs and learn new facts. Because of our assumption that ADL and SDL are shared and IDL concepts are specialisations, they can easily be placed in the taxonomy. A simple form of learning is incorporated this way.

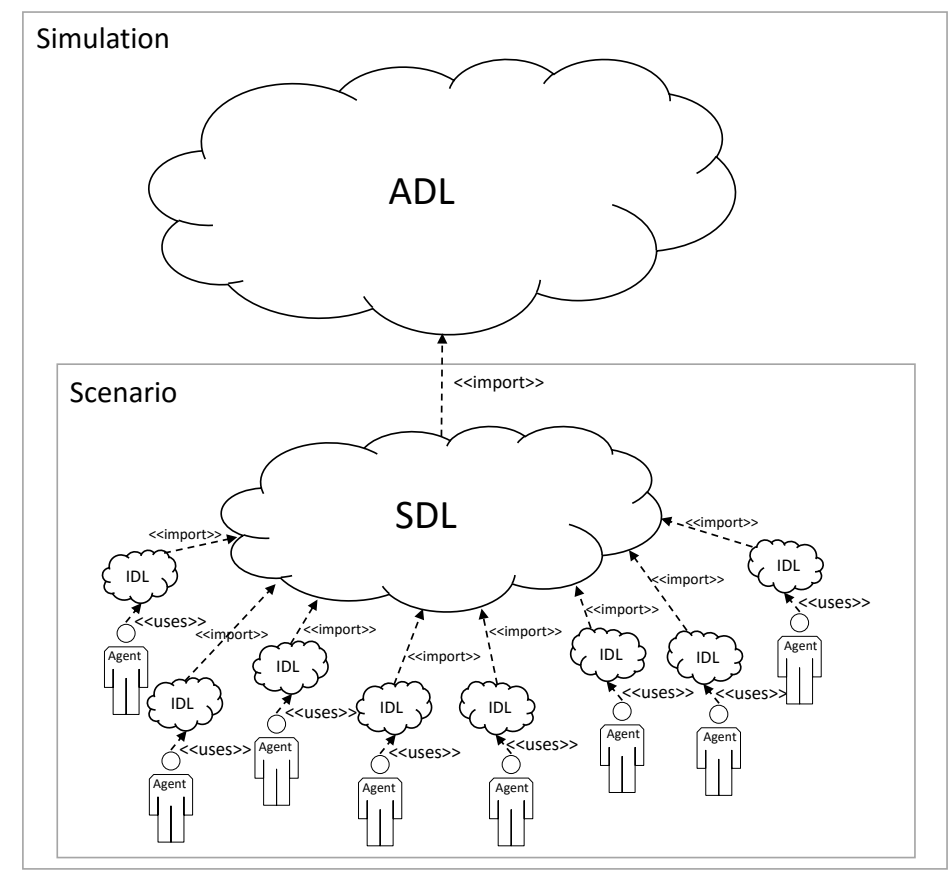

Figure 2: Three layered ontology 
2.5 We developed an AbstractOWLAgent Java class that describes fundamental elements of an OWL-BDI agent required to participate in AGADE simulations. References and methods to maintain ontologies and trigger plans are implemented here. Subclasses of AbstractOWLAgent are on the level of SDL and specify more concrete aspects of an agent. Each agent is linked to an IDL in which its individual behaviour is defined. AGADE uses the inference mechanisms of the ontology (implemented in a reasoner) to determine the actions an agent performs to reach its goals (i.e. desires). In compliance with the Jadex framework, possible individual actions of an agent have to be implemented as plans within the Java code that implements the agent. Jadex allows these plans to either be represented as methods inside the Java class that implements the agent or alternatively as plain Java classes which have to provide a so called plan body method. For reasons of flexibility and reusability we decided to code plans as Java classes. The Java classes annotated as plans have a corresponding member of concept AgentAction in the ontology. These links make facts and rules of the ontology accessible to the agents in the Java code. The object property nextAgentAction (with domain Person, which is basically equivalent to the set of agents, and range AgentAction) together with a rule determines how the agent decides which plan to chose next. The next agent actions are periodically triggered by the round-based management of AGADE.

2.6 On an abstract level AGADE (see Figure 3 comprises two different kinds of BDI agents: a director type agent that acts as a specifically marked coordinator for the simulation triggering the beginning of each new round and a participant type that comprises any kind of agent participating in the simulation (i.e. consumer and seller).

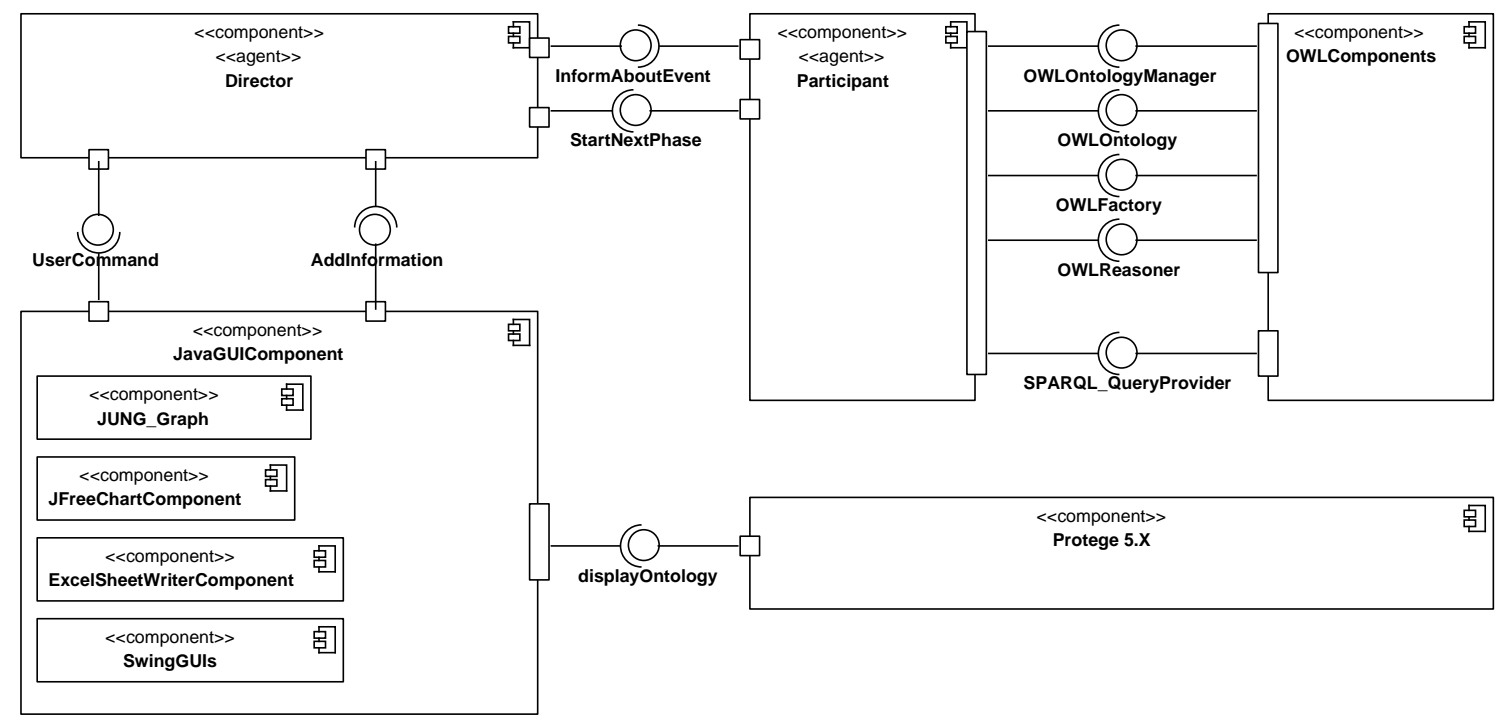

Figure 3: Abstract view on the AGADE architecture.

2.7 Each round (time step) of the simulation is structured into four phases with defined functionality and integration into the BDI paradigm:

Control phase The director processes commands issued by the user during execution of the last round. GUI components are updated.

Calculation phase The director tells each agent to make necessary calculations to update its internal state.

Socialisation phase Agents update their mutual relations and possibly build new connections to other agents.

Acting phase The next agent actions are triggered depending on the individual state of the agent and the results of rule evaluation.

2.8 The ontology based belief base leads to a very flexible architecture, because important aspects of the agent do not have to be coded statically any more but may instead be expressed in the rules of the ontology. In the next section we describe a typical scenario and demonstrate how it can be implemented using AGADE. 


\section{Scenario}

3.1 In this section we demonstrate how the three layer ontology architecture can be applied and how user interaction enables the use of AGADE within business games. The following scenario is used to validate the three layer ontology model.

\section{Summary}

3.2 Kiesling et. al. describe an agent-based model for the diffusion of a second generation biofuel product into the Austrian market (Kiesling et al. 2010). High initial investments in infrastructure are necessary to launch biofuel into the fuel market, therefore considerable financial resources are at stake before a sufficiently large share of the market can be conquered. This is why the simulation focusses on the crucial questions of whether biofuel will be accepted by the market at all and to what extent consumers will actually use biofuel abandoning their traditional choice. Kiesling prefers an agent-based model over alternative approaches because existing mathematical models "do not distinguish between the individual characteristics of consumers and thus neglect consumers' heterogeneity with regard to preferences and behaviour".

3.3 In the simulated fuel market each product is characterised along multiple dimensions using a variety of attributes such as price, quality, or environmental friendliness. Consumers estimate product characteristics based on their limited individual level of information which is either fed by personal experience or by communication with other individuals or advertising. Each agent represents a single consumer and acts in a social structure which is constructed according to network theoretic algorithms e.g. preferential attachment or the Watts-Strogatzmodel (Scott2012). Kiesling characterises the participating agents by a number of individual parameters among which we find the so called innovative threshold. This is used to model individual innovativeness meaning the willingness to adopt innovations e.g. prefer new products or new brands over established ones. Even if they would profit from using biofuel instead of conventional fuel, consumers may still refuse to purchase it just because it is simply too new. They rather wait for others and their experiences. This fact is modelled by means of the innovative threshold. According to Roger's model of innovativeness (2003), this threshold can be used to categorise the consumers into innovators, early adopters, early majority, late majority, and laggards. More parameters are available that describe the role of the individual as a target of communication and its individual behaviour as a consumer. Each agent keeps an information level (short: info( $p, a)$, for product $p$ and agent $a$ ) that may increase through communication with fellow agents or by being target of marketing activities. This value automatically decreases over time as information is obsolescing if not updated. The driving behaviour of an agent is modelled through a parameter that determines fuel consumption and in consequence the frequency of stops at the filling station. Furthermore, the agent has a utility threshold that describes the level of utility a product must have to be considered as a valid alternative. Product quality is modelled by the product quality value (short: $p p q(p, a)$, for product $p$ and agent $a$ ) which reflects consumer experiences with the product. The utility value describes the subjective utility a consumer ascribes to a product and determines product selection.

3.4 Agents decide to buy a new product only if the utility value of that product is higher than the utility threshold of the agent. The utility value of a product $p$ for an agent $a$ is calculated with the following utility function:

$$
u_{t}(a, p)=\left(1-\operatorname{price}_{t}(p)\right) \cdot w_{1, t}^{a}+\operatorname{price}_{t}(p) \cdot w_{2, t}^{a}+\operatorname{ppq}_{t}(p, a) \cdot w_{3, t}^{a}+\text { info }_{t}(p, a) \cdot w_{4, t}^{a}
$$

where $w_{i}, t^{a} \geq 0$ and $\sum_{i}=1^{4} w_{i, t}^{a}=1$ for each agent $a$ and each time period $t$. For brevity we omit the index $t$ meaning that the proposition holds for each value of $t$. The weights are related to the price $\left(w_{1}\right.$ and $\left.w_{2}\right)$, the preference for high quality $\left(w_{3}\right)$, and the readiness to buy renewable energy $\left(w_{4}\right)$ which depends on information.

3.5 The set of consumers is partitioned into four segments which is reflected in the four weights of the utility function:

- price-sensitive consumers with emphasis on low prices (largest segment, 70 percent) have a high value for $w_{1}, w_{2}=0$ and low values for $w_{3}$ and $w_{4}$

- consumers with focus on high-quality (15 percent) high value for $w_{3}, w_{2}=0$ and low values for $w_{1}$ and $w_{4}$

- eco-consumers for which price or quality are less important than environmental friendliness (10 percent) high value for $w_{4}, w_{2}=0$ and low values for $w_{1}$ and $w_{3}$ 
- snob buyers looking for exclusive products taking high prices as a signal for high quality (smallest segment, 5 percent) have a high value for $w_{2}, w_{1}=0$ and low values for $w_{3}$ and $w_{4}$

3.6 Note that the sizes of the segments are estimations based on the description given by Kiesling. Agents are assigned randomly to the segments.

3.7 The dynamics of the permeation process of biofuel in the fuel market is modelled with three types of events that can occur during a simulation: communication events, need events and experience events. The events are either triggered stochastically or periodically depending on their nature.

Communication events Communication events are generated on the set of agents and are used to model the diffusion of information in the agent society. An agent is selected randomly and then decides again at random which of its relations it chooses to communicate with. The number of such mutual communication events in a single round of the simulation is proportional to the number of neighbours of the agent chosen. During the communication the agent with the lower information level adjusts this value according to that of the communication counterpart following an influence factor defined on the connection in the social network weighing the influence.

Need events A need event occurs whenever an agent encounters a deficit triggering a purchasing process. This deficit is indicated when the fuel level falls below the fuel threshold of the agent. If the agent does not have any knowledge about biofuel at all, conventional fuel is bought. Otherwise, the utility value of biofuel is calculated and if the resulting value lies above the individual utility threshold, the agent chooses biofuel.

Experience events In between two need events an experience event occurs. Experience events model personal experiences with a product by directly updating the quality value of the product.

3.8 Marketing activities are scheduled periodically to introduce new products and to spread information about established products. Agents are selected randomly and have their information level updated. Note that besides personal communication which follows the structure of the social environment (word of mouth and opinion leadership) marketing activities are the second means to spread knowledge in the community.

\section{Fuel market ontology}

3.9 While we can reuse the abstract domain layer (ADL) of our previous simulations (i.e. mobile phone market, Farrenkopf et al. 2014, 2015 without changes, the specific domain layer ontology (SDL) has to be adapted to describe the fuel market with its products and specific elements (see Figure 4. Furthermore, specific aspects of agent behaviour have to be implemented in the individual ontologies of seller and consumer. In this process abstract concepts have to be specialised by subconcepts (e.g. Person gets subconcepts FuelSeller and FuelConsumer while Fuel becomes a subconcept of Product which again is a subconcept of Item). The four consumer segments (see Figure 4 are modelled as a value partition i.e. the set of consumers is the union of the four disjoint subsets Price-sensitiveConsumer, Quality-seekingConsumer, Eco-Consumer and Snob-buyer that are again modelled as subconcepts of Consumer (taken from SDL) and ValuePartition (taken from ADL). Relevant variables such as fuel level, travel behaviour, and utility value are modelled as data properties (see Figure 5) having the respective concepts as range. 


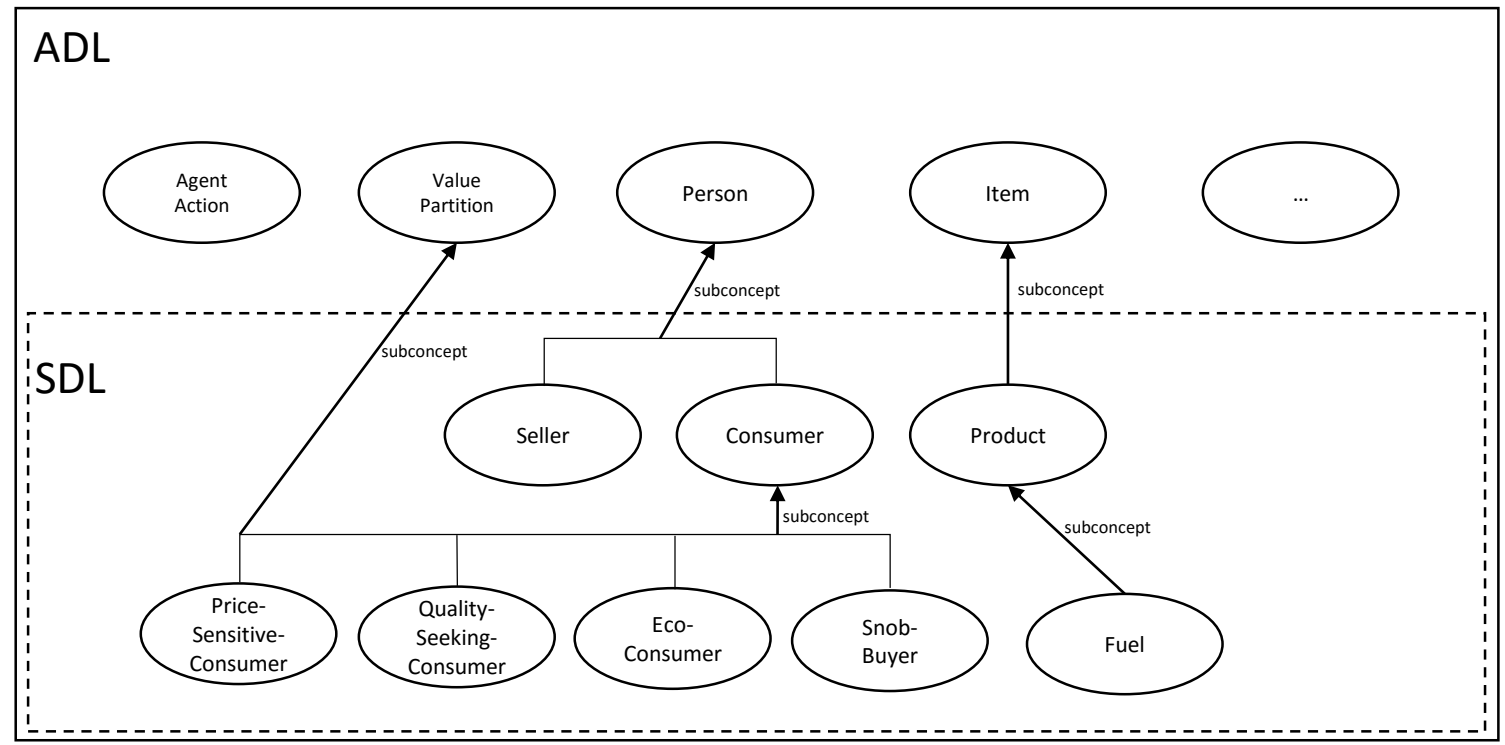

Figure 4: Subconcepts of the specific domain layer.

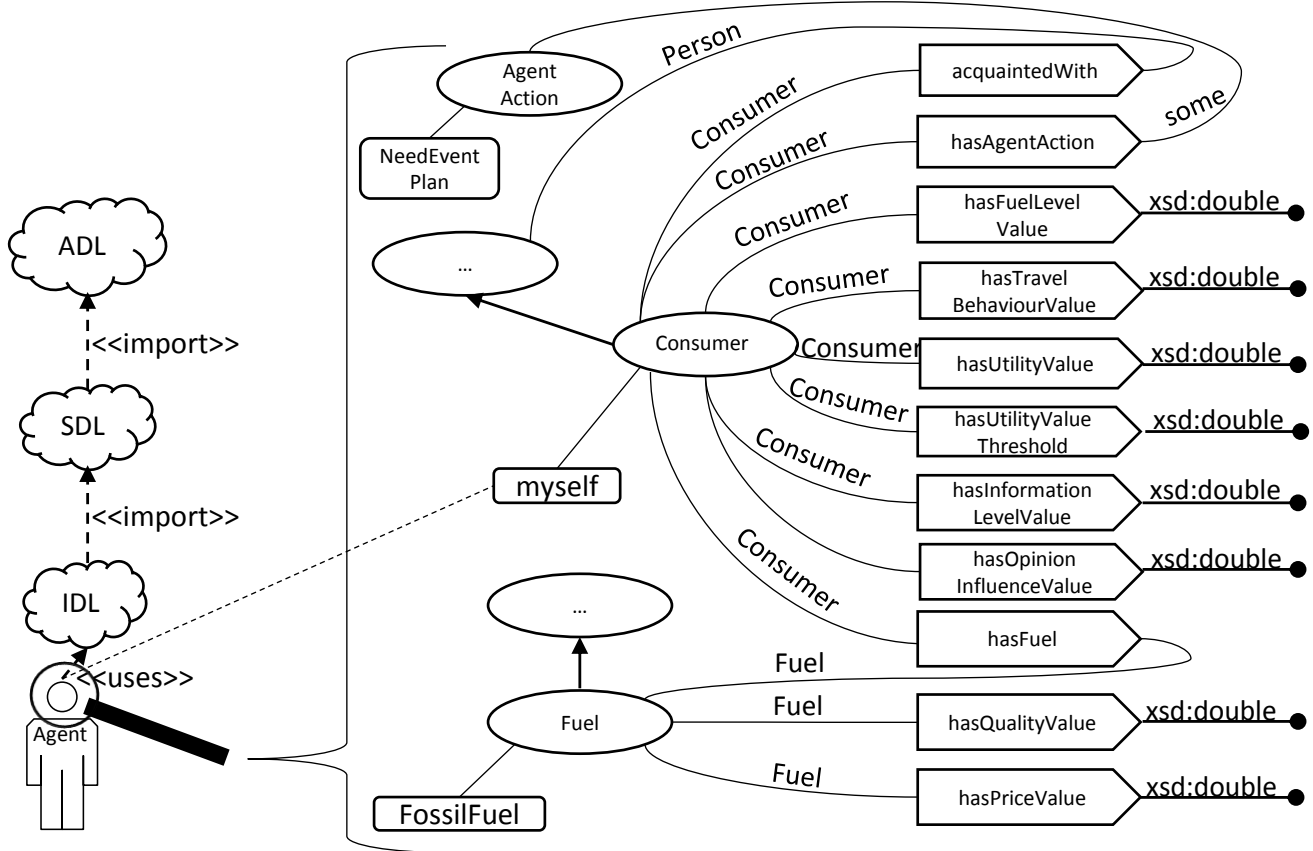

Figure 5: Biofuel individual domain layer.

3.10 Besides containing the concepts of the market domain ontology, the IDLs define all relevant facts to model the state as well as the individual behaviour of the agents making extensive use of SWRL rules. The following example shows an SWRL rule that is used to determine the appropriate plan of a consumer agent for the next simulation round. If the fuellevelvalue of consumer falls below its individual threshold, a need event is triggered i.e. the next agent action will be set to NeedEventPlan by using ontology reasoning techniques. This plan implements the process in which the product is chosen.

Person(myself), hasFuelLevel(myself, ?hfl),

hasFuelLevelThreshold(myself, ?hflt), lessThan $(? h f l, ? h f l t)$

$\rightarrow$ hasNextAction(myself, NeedEventPlan) 
3.11 Note that although SWRL provides math built-ins that enable OWL reasoners to perform simple mathematical operations, we implemented all numerical computations in Java and made them accessible to the ontology through method calls. We preferred that, because the support of SWRL built-ins is limited and may cause SWRL rules to loose decideability, which will let the reasoning mechanisms fail (Feng et al. 2010).

3.12 Agents communicate with other agents (e.g. they exchange information about product details) and this communication refers to knowledge items that belong to the IDL layer. For this reason, agents can exchange information which contains concepts that may be totally new for the receiving agent. The agent may then add new facts (e.g. information level of biofuel or price value) acquired through this information exchange into its belief base. When incorporating a new concept into its IDL (e.g. introducing biofuel through a marketing campaign), the agent can add the concept into its IDL in an appropriate way because of sharing SDL and ADL.

\section{Parameters and results}

3.13 Agents and their relations form a small world network of 30000 consumer agents and 1 seller agent built with preferential attachment according to the Barabási algorithm which builds the network iteratively by adding nodes to an initial graph. The overall number of nodes was chosen due to limitations of the available hardware (two quad core CPUs and 256GB RAM). We modified the original algorithm so that the resulting network is directed. The number of connections of a new node was set to 225 . Any node that is connected to more than 3000 nodes is considered a hub. Due to the parameter settings of the network construction, approximately 1 percent of agents are hubs. The weight of the edges connecting nodes to a hub is randomly set to an opinion influence value between 0.8 and 1 . This lets hubs act as opinion leaders who strongly influence buying decisions of other consumers through communication events. Experience events for an agent occur randomly in each round after the first time the agent has bought biofuel.

3.14 The travel behaviour of an agent is represented by a stochastic variable which is initially calculated for each agent and based on a normal distribution with a mean of 0.3 and a standard deviation of 0.05 . In each time period the fuel level is reduced by this value. The individual threshold that leads to a stop at the filling station (see above fuel level threshold) is set to a random number between 0 (exclusive) and 0.20 (inclusive). The price value of biofuel is set to $1.20 €$ and the price value of fossil fuel is set to $1.00 €$.

3.15 Initially, fossil fuel is the only fuel consumers can buy. At the beginning of the simulation they are not even aware that there is an alternative product such as biofuel. When biofuel is launched into the market, the seller agent initiates marketing activities to spread information about the new product. Agents add this new information to their private IDL. The timing of the product launch can be chosen arbitrarily. For comparison reasons with Kiesling, we set that point in time to round 85 . However, this has no significant effect on the curve progression, but only shifts the graph on the time axis.

3.16 Marketing activities focus on increasing the information level of each agent within reach of the activity. A marketing activity contains the variable information content value. This is set to a random value between 0 and 1 and models the amount of information that is carried by the activity. The amount of marketing activities is limited to $1 \%$ of the overall number of participating agents in each period. Whenever an agent is reached by a marketing activity, the information content value is added to biofuel information level of the agent. If an agent is affected by more than one marketing activity in one time period, the maximum of the information content values of all activities will be added. As described above the decision of buying biofuel instead of fossil fuel finally depends on the individual utility values and the individual thresholds.

\section{Conclusion}

3.17 The time horizon was set to 400 rounds (i.e. time periods) and a total of 100 runs with varying random seeds. As expected, the results (see Figure 6 and Figure 7 are very similar to Kiesling's results. Differences occur because not all of Kiesling's parameter settings were published with exact values. Reasonable assumptions were made here. The results prove the principal applicability of our concept. Certainly, this scenario can be reproduced with other agent simulation frameworks likewise, but AGADE is more flexible and versatile in modelling different agent behaviour. Important aspects of the agents do not have to be coded statically any more but can be declared with rules. We will now focus on the perspective of using AGADE as a business game. Users will be enabled to selectively change parameter settings and explore the resulting effects thus ultimately analysing the problem space of the scenario by setting up a hypothesis and then run simulations against it. A learning circle can be established by either accepting or rejecting the hypothesis leading to further refinements of the assumptions or alternative claims. This point of view confirms to the theory of Firestone and McElroy which 
states that organisations and individuals acquire knowledge through what they call decision execution cycles (DEC) in which actions are based upon knowledge claims, which represent untested assumptions. The effect of the actions is then compared to the expectations and a possible gap is closed by adjusting the initial knowledge claim according to the results observed (Firestone 2003). The theory is highly influenced by Karl Popper's epistemology (1979).

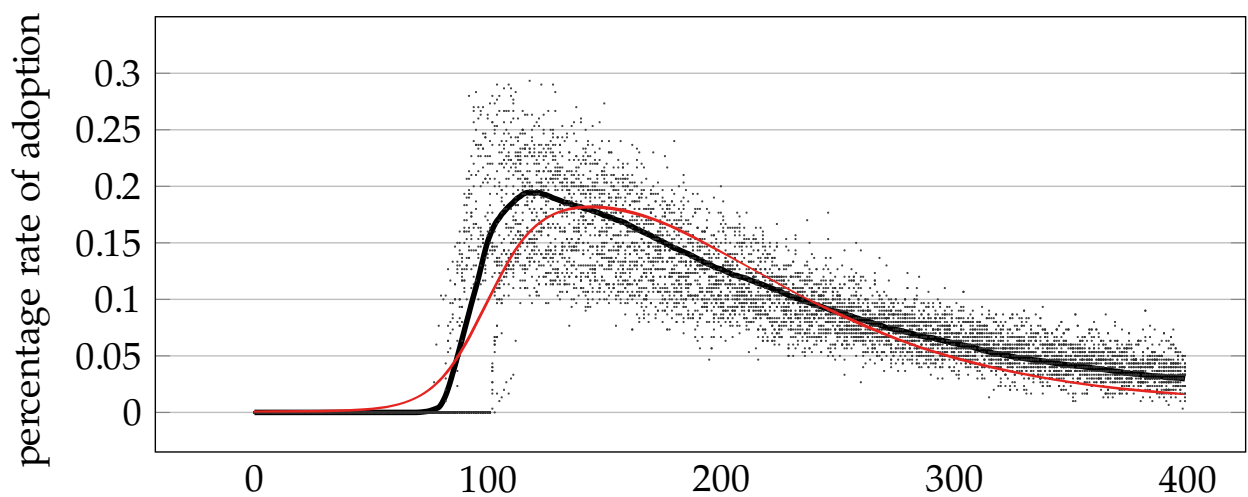

Figure 6: Biofuel adoption curve (points mark results of single simulation runs, black line indicates averages by AGADE and the red line is the average line published by Kiesling)

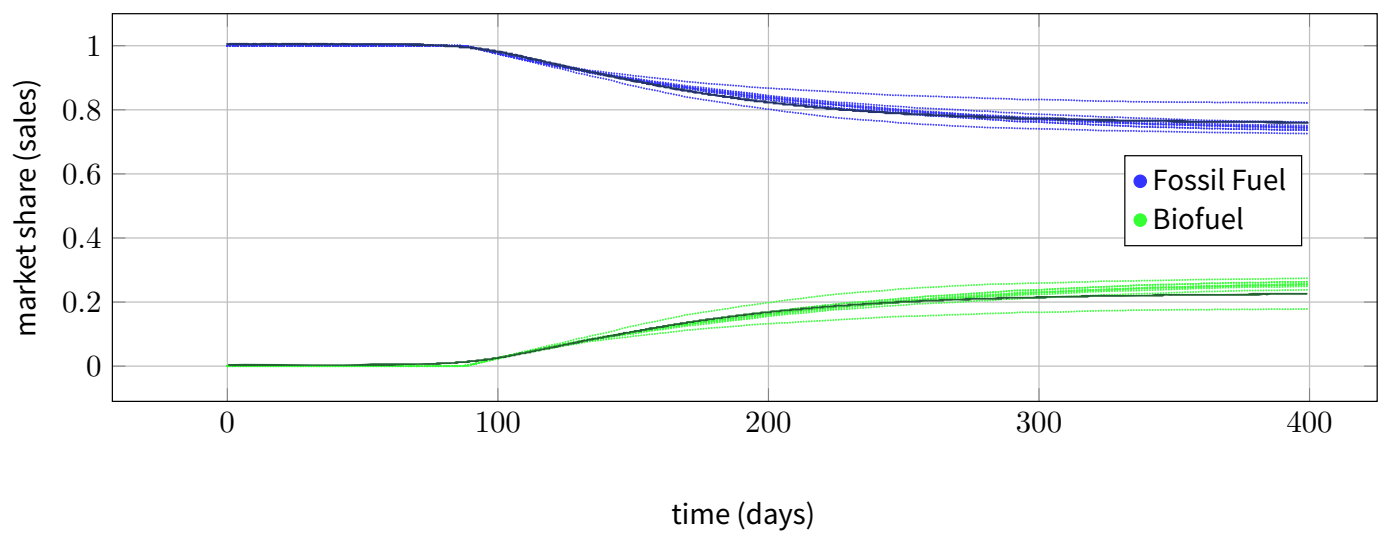

Figure 7: Unit sales volume market share (data generated by AGADE) 


\section{Business game considerations}

3.18 Business games are a specific subspecies of what is called a serious game. The concept combines business simulations and games to support management and entrepreneurial training (Baldissin et al. 2013, p. 3). Business games are used as an educational mean to go beyond mere theoretical contemplation and to simulate practice. They provide students with the opportunity to develop decision-making skills and improve confidence in situations of risk and uncertainty through the simulation of management practices across the enterprise in ever more complex real-life contexts.

3.19 We have described a scenario where marketing activities are crucial for placing a new product into a saturated market. Here, challenges certainly lie in a limited marketing budget and the unpredictability of the effects of applied marketing means. The overall goal is of course to increase revenues. In this case business games will train the ability to plan effective campaigns that make optimal use of a given budget.

3.20 Providing a user interface through which parameter settings can be modified during the simulation, AGADE lets users intervene in the simulations. Users can experiment with various settings and investigate strategies that may accelerate the adoption of biofuel by the market and thus increase the market share. After each period users will receive feedback that describes the evolution of the market and can then draw conclusions. Users can analyse and contemplate results and decide what to do in the next period. Note that existing equation-based simulations usually do not present results to participants with real-time animations, as they can only display the final output of equations at the end of each round. An example for a possible action is cutting consumer prices which of course will have an impact on the sales figures and on the revenues. This impact will immediately be reflected by the system and the calculations of the next round. A possible hypothesis to be tested is 'If you win opinion leaders, you will convince their community as well'. This hypothesis can be tested by simulating target marketing (Weinstein 2013, pp. 133-154), which can be done by identifying hubs in the social network and observe how word-of-mouth mechanisms let information diffuse through the network. Social network analysis can identify hubs by simply looking for agents with a high number of connections to fellow agents. As finding opinion leaders in real world communities is difficult and expensive, it is well worth the effort to examine the effects of opinion leaders in a business simulation before starting to locate them in the community. Without simulations this would have to be done with field experimentation (compare Coleman et al. 1966).

3.21 The simulation itself can be controlled through the GUI displayed in Figure 8 . A simulation can be halted between any two rounds by clicking the GUI control buttons located at the top so that further inspection of the current state of affairs is possible. Data describing crucial aspects of the current simulation is displayed continuously. The right hand side of the screen is a visualisation of the social structure formed by all participating agents. The vertices of the graph represent the agents using different shapes for different agent types. Size and colour of the vertices can be used to display additional information on the respective agent (e.g. individual state). The edges between the vertices visualise details about a relation and are labelled with the respective relation indices (e.g. opinion influence level). Importantly, the social graph can be used to inspect the very mind of each agent, as it allows to take a look at the current ontology state belonging to an agent of interest. The intention of a consumer agent to refuel its car is indicated by setting the colour of the respective vertex to red. The user interface control is displayed at the start of the simulation and then after a predefined number of steps (see Figure 9]. At each stop the user can specify the amount of money to be invested in marketing activities in the next rounds and select a marketing strategy (i.e. target marketing). Beyond that, the price of the product under observation (here biofuel) can be adjusted. The feature that the social graph is continuously updated visualises the dynamics of information diffusion. 


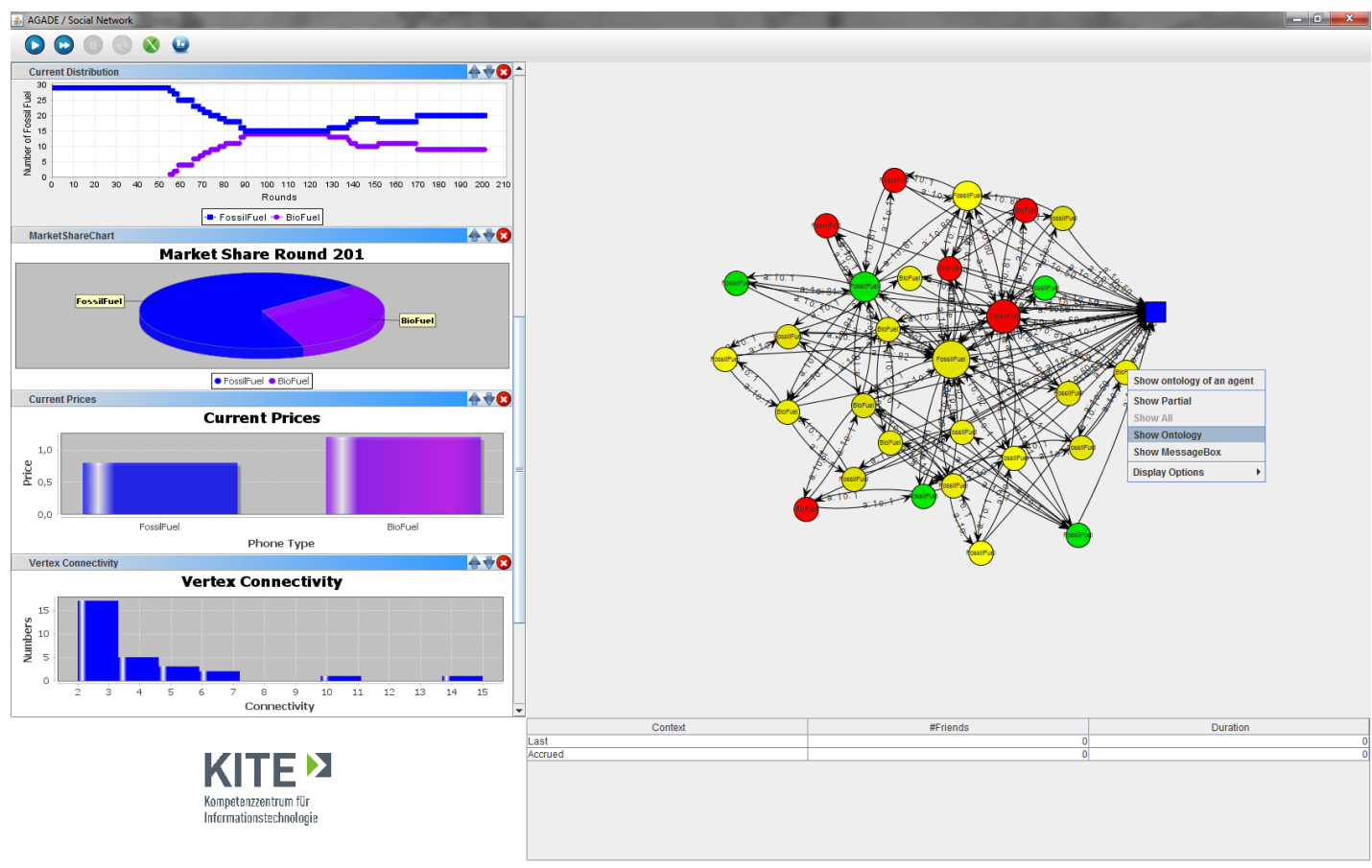

Figure 8: The main GUI displayed during a simulation process.

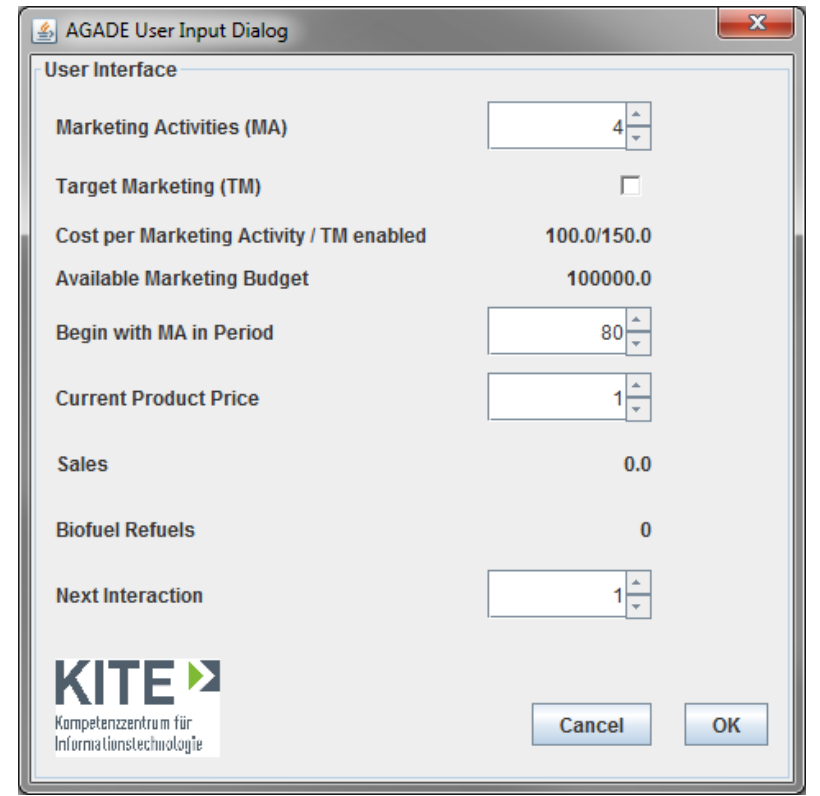

Figure 9: AGADE user input dialog.

3.22 We conducted an experiment with two restricted groups of students in the classroom (10 first year bachelor's program students and 20 second year master's program students, both business informatics) to investigate how simulation settings respond to business decisions. Two groups of students with different levels of knowledge of the topic marketing and with different levels of experience in the use of business games were selected. While the first year bachelor students had never actively taken part in business games in the classroom before, the master program students already had used and reflected their use and effect (e.g. Topsim ${ }^{1}$ and Simultrain ${ }^{2}$ ). The experiment was divided into three phases: instructions, pre-experiment test and post-experiment test. The instructions provided in the first phase described the purpose of the experiment and gave instructions on how to use AGADE. The pre-experiment test consisted of questions about the law of demand and supply and other questions regarding marketing effects. The intention here was to establish a mental preparation of the test persons towards the scenario of the game and eventually to assess the subjects' knowledge about particular 
economic concepts. The post-experiment was a questionnaire in which the reaction of the subjects and the user experience made after running the simulation was to be measured. We used the usual 5-point Likert scale from strongly disagree to strongly agree. The overall duration of the experiment was 90 minutes. Table 1 shows the list of questions used.

Table 1: Survey questions

\begin{tabular}{ll}
\hline ID & Question \\
\hline q1 & The simulation course meets my expectations \\
q2 & The simulation appears to be realistic \\
q3 & Playing the simulation gave me new insights into market dynamics \\
q4 & The simulation explained possible effects of marketing activities \\
q5 & The simulation responds on my decisions \\
q6 & The scenario was easily comprehensible \\
q7 & The scenario was complex \\
q8 & The scenario is suitable for a business game \\
q9 & The scenario is suitable for a marketing training \\
q10 & My theoretical marketing knowledge was adequate \\
q11 & Practical relevance \\
q12 & Encourages teamwork \\
\hline
\end{tabular}

3.23 In this section, we analyse the results of the questionnaire in Table 1 of both student groups. Figure 10 compares the mean values of the answers for both groups. Both student groups produced similar values in their answers with the exception of question 10. The significant lower value of the question 10 is due to the fact that it was a first year student group which had not visited a marketing lecture yet. Based on these results, we claim that the simulation responds to the decisions made by the users in a manner that is expected (see questions 1, 2 and 5). The figure shows that the scenario is principally applicable to be used for business simulations (q3, q4, q6, q7, $q 8, q 9, q 11, q 12)$. Despite the different levels of knowledge of both groups there are only slight differences in the results. Note that both groups were made familiar with basic marketing theory. All in all, the simulation of the consumer market was accepted as a valid model of reality and shows the applicability of the concept of using ontology based BDI agents to model consumer behaviour and communication.

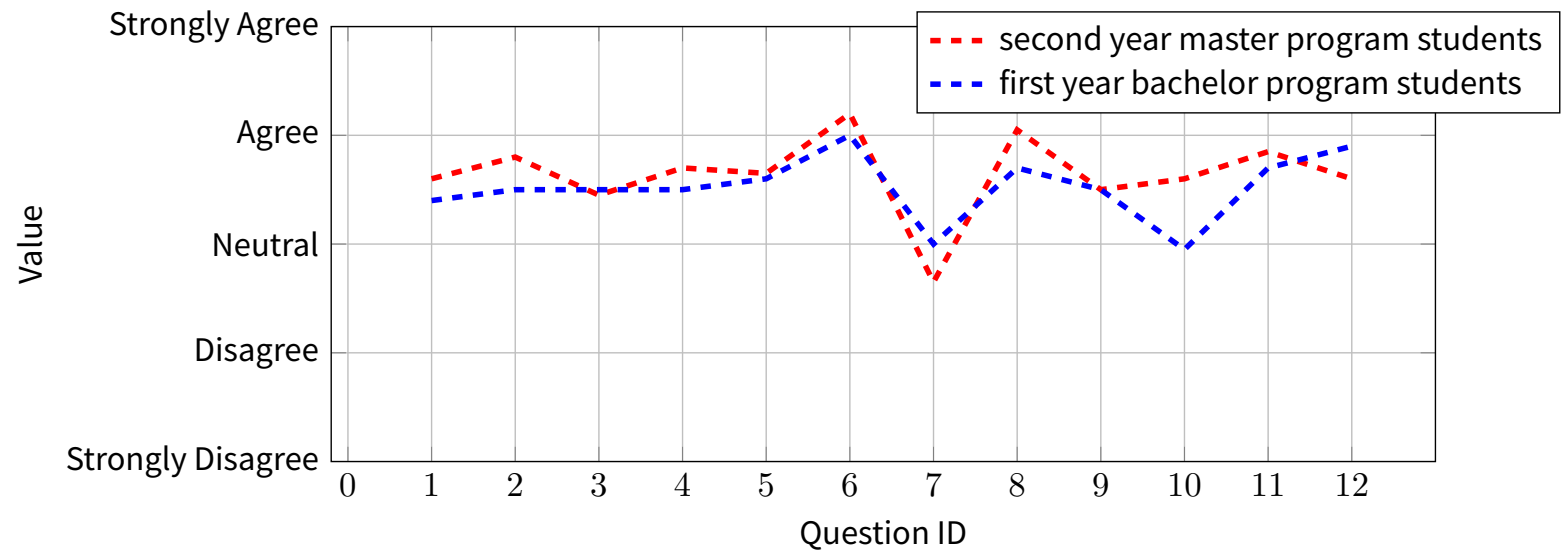

Figure 10: Results of the questionnaire

\section{Conclusion and Future Work}

4.1 Using ontologies to model agents can have a significant impact on the way agent oriented programming and software simulations work. With AGADE, we show how multi-agent based system and semantic technologies are used to model individuals participating in a dynamic market environment. A three-layer ontology allows agents to share knowledge and create a basic common understanding of their environment while enabling reuse of fundamental concepts and simple learning mechanisms. Modelling and use of overall individual behaviour and decision processes in simulations for different scenarios which can be used in business games is demonstrated. 
4.2 Assumptions about consumer behaviour, decision making, and effects of social communities can also be put into the models. This leads to a basis for running business games in which decision making and its effects can be simulated. The study of a scenario in which a new product is launched into a saturated market shows the potential of the approach to be applied in more complex contexts.

4.3 Future research will complete the set of implemented behavioural patterns and make them available in business games. Furthermore, we consider a domain specific language designed to both accelerate and facilitate the development of powerful AGADE components. The degree of reuse that can be achieved will be investigated and formalised.

\section{Notes}

http://www.topsim.com

2 http://www.simultrain.com

\section{References}

Baader, F. (2003). The description logic handbook: theory, implementation, and applications. Cambridge university press

Baldissin, N., Bettiol, S., Magrin, S. \& Nonino, F. (2013). Business game-based learning in management education. Business Game srl

Bratman, M. (1999). Intention, plans, and practical reason. Stanford and Calif: Center for the Study of Language and Information

Coleman, J., Katz, E. \& Menzel, H. (1966). Columbia university. bureau of applied social research: Medical innovation: a diffusion study. Advanced Studyies in Sociology, Bobbs-Merrill Co

Farrenkopf, T., Guckert, M., Hoffmann, B. \& Urquhart, N. (2014). AGADE - how individual guidance leads to group behaviour and how this can be simulated. In J. P. Müller, M. Weyrich \& A. L. C. Bazzan (Eds.), Multiagent System Technologies - 12th German Conference, MATES 2014, Stuttgart, Germany, September 23-25, 2014. Proceedings, vol. 8732 of Lecture Notes in Computer Science, (pp. 234-250). Springer. doi:10.1007/978-3-319-11584-9_16

Farrenkopf, T., Guckert, M. \& Urquhart, N. (2015). AGADE using personal preferences and world knowledge to model agent behaviour. In Y. Demazeau, K. S. Decker, J. B. Pérez \& F. de la Prieta (Eds.), Advances in Practical Applications of Agents, Multi-Agent Systems, and Sustainability: The PAAMS Collection - 13th International Conference, PAAMS 2015, Salamanca, Spain, June 3-4, 2015, Proceedings, vol. 9086 of Lecture Notes in Computer Science, (pp. 93-106). Springer. doi:10.1007/978-3-319-18944-4_8

Feng, Y., Liu, Y., Li, Y.-F. \& Zhang, D. (2010). Discovering anomalies in semantic web rules. In Proceedings of the 2010 Fourth International Conference on Secure Software Integration and Reliability Improvement, SSIRI '10, (pp. 33-42). Washington, DC, USA: IEEE Computer Society. doi:10.1109/SSIRI.2010.26

Firestone, M., J.M.; McElroy (2003). Key Issues in the New Knowledge Management. Butterworth Heinemann

Forrester, J. (1990). Principles of systems. System dynamics series. Productivity Press

Gros, C. (2008). Complex and adaptive dynamical systems: a primer. Springer Complexity. Berlin, Heidelberg: Springer

Gruber, T. R. (1993). A translation approach to portable ontology specifications. Knowledge Acquisition, 5(2), 199-220

Hadzic, M. (2009). Ontology-based multi-agent systems, vol. v. 219 of Studies in computational intelligence. Berlin: Springer

Horridge, M. \& Bechhofer, S. (2011). The owl api: A java api for owl ontologies. Semantic Web, 2(1), 11-21 
Horrocks, I., Patel-Schneider, P. F., Boley, H., Tabet, S., Grosofand, B. \& Dean, M. (2004). SWRL: A semantic web rule language combining OWL and RuleML. W3C Member Submission. Last access on October 2016 at: http://www.w3.org/Submission/SWRL/

Kaynak, E., Wolfe, J. \& Keys, J. (2012). Business Simulations, Games, and Experiential Learning in International Business Education. Taylor \& Francis

Kiesling, E., Günther, M., Stummer, C., Vetschera, R. \& Wakolbinger, L. M. (2010). A spatial simulation model for the diffusion of a novel biofuel on the austrian market. In A. Bargiela, S. Ali, D. Crowley \& E. Kerckhoffs (Eds.), ECMS, Proceedings of the 24th European Conference on Modelling and Simulation (ECMS 2010), (pp. 41-49)

Popper, K. R. (1979). Objective Knowledge: An Evolutionary Approach. Oxford: Clarendon Press, revised edn.

Rogers, E. (2003). Diffusion of Innovations, 5th Edition. Free Press

Scott, J. (2012). Social Network Analysis. SAGE Publications

W3C OWL Working Group (2012). OWL 2 Web Ontology Language: Document Overview. W3C Recommendation. Available at http://www .w3.org/TR/2012/REC-ow12-overview-20121211/

Weinstein, A. (2013). Handbook of Market Segmentation: Strategic Targeting for Business and Technology Firms, Third Edition. Taylor \& Francis

Wilensky, U. \& Rand, W. (2015). Introduction to agent-based modeling: Modeling natural, social, and engineered complex systems with NetLogo. Cambridge, Mass.: The MIT Press 